\title{
The characteristics of blood glucose fluctuations in patients with fulminant type 1 diabetes mellitus in the stable stage
}

Jie Wang ${ }^{1, *}$, Bing-Li Liu', *, Zheng Li', Hui-Qin Li', Rui Sun', Yun $\mathrm{Hu}^{1}$, Kok-Onn Lee², Lei Ye ${ }^{3}$, Xiao-Fei Su', Jian-Hua Ma'

\begin{abstract}
Objective: The aim was to characterize blood glucose fluctuations in patients with fulminant type 1 diabetes (FT1DM) at the stable stage using continuous blood glucose monitoring systems (CGMSs). Subjects and methods: Ten patients with FT1DM and 20 patients with classic type 1 diabetes mellitus (T1DM) (the control group) were monitored using CGMSs for 72 hours. Results: The CGMS data showed that the mean blood glucose (MBG), the standard deviation of the blood glucose (SDBG), the mean amplitude glycemic excursions (MAGE), the blood glucose areas and the percentages of blood glucose levels below $13.9 \mathrm{mmol} / \mathrm{L}$ were similar between the two groups. However, the percentage of blood glucose levels below $3.9 \mathrm{mmol} / \mathrm{L}$ was significantly higher in the FT1DM group compared to the T1DM group ( $<$ 0.05). The minimum (Min) blood glucose level in the FT1DM group was significantly lower than that of theT1DM group $(p<0.05)$. Patients with FT1DM had severe dysfunction of the islet beta cells and alpha cells compared to patients with T1DM, as indicated by lower C-peptide values and higher glucagon/C-peptide values. Conclusion: In conclusion, patients with FT1DM at the stable stage were more prone to hypoglycemic episodes as recorded by CGMSs, and they had a greater association with severe dysfunction of both the beta and alpha islet cells compared to patients with T1DM. Arch Endocrinol Metab. 2018;62(6):585-90
\end{abstract}

Keywords

Fulminant type 1 diabetes; type 1 diabetes; continuous glucose monitoring system (CGMS)
${ }^{1}$ Department of Endocrinology, Nanjing First Hospital, Nanjing Medical University, Nanjing, China ${ }^{2}$ Division of Endocrinology, Department of Medicine, National University of Singapore, Singapore ${ }^{3}$ National Heart Research Institute Singapore, National Heart Centre Singapore, Singapore

* Contributions were equal to those of first author

Correspondence to: Jian-Hua Ma Department of Endocrinology Nanjing Medical University Affiliated Nanjing Hospital 210006 - Nanjing China majianhua@china.com Xiao-Fei Su

Department of Endocrinology Nanjing Medical University Affiliated Nanjing Hospital 210006 - Nanjing China suxiaofeifei@126.com

Received on Jan/12/2018 Accepted on Jun/14/2018

DOI: 10.20945/2359-3997000000082

\section{INTRODUCTION}

$\mathrm{T}$ ype 1 diabetes mellitus (TIDM) is characterized by insulin deficiency and the destruction of pancreatic beta cells. It is mainly classified into classic TIDM and fulminant type 1 diabetes mellitus (FTIDM). FTIDM is a special subtype of type 1 diabetes first proposed by Imagawa and cols. in 2000 (1). FTIDM is characterized by an abrupt onset, dangerous conditions, ketosis or ketoacidosis at the time of diagnosis, high blood glucose levels, lower glycosylated hemoglobin (HbAlc) levels, serious destruction of pancreatic beta cells, and negative islet-related autoantibodies, such as insulin antibodies, anti-GAD antibodies (GADAb), and insulin autoantibodies (IAA). In addition, most patients with FTIDM report having had symptoms of influenza or gastrointestinal symptoms prior to onset (2). Recently, reports on FTIDM have increased, especially in East Asia (2-6). However, detailed characteristics of glucose fluctuations in patients with FTIDM at the stable stage are unclear.
The current study aimed to utilize CGMSs to characterize blood glucose fluctuations in patients with FTIDM at the stable stage.

\section{SUBJECTS AND METHODS}

\section{Subjects}

This was a retrospective study. We identified 10 patients with FTIDM at the stable stage and 20 patients with classic TIDM as control patients from Jan. 2012 to Dec. 2015 at Nanjing First Hospital, Nanjing, Jiangsu, China. The FTIDM group included 6 female and 4 male patients, and their mean history of TIDM was $7.20 \pm 5.83$ years. All of the patients with FTIDM met the diagnostic criteria proposed by Imagawa: 1) ketosis or ketoacidosis that occurred in a high glucose state, usually less than 1 week; 2 ) plasma glucose levels $>16 \mathrm{mmol} / \mathrm{L}$ ( $>288 \mathrm{mg} / \mathrm{dL}$ ) and HbAlc levels $<8.7 \%$ in newly diagnosed patients; 3 ) excretion of urinary 
C-peptide $<10 \mathrm{ug} / \mathrm{dL}$ with a fasting plasma C-peptide $(\mathrm{C}-\mathrm{P})<0.1 \mathrm{nmol} / \mathrm{L}(0.3 \mathrm{ug} / \mathrm{L})$ and stimulation plasma C-peptide (after a meal or glucagon injection) $<0.17 \mathrm{nmol} / \mathrm{L}(0.5 \mu \mathrm{g} / \mathrm{L})(7)$. The TIDM group included 8 female and 12 male patients, and their mean history of TIDM was $9.60 \pm 8.40$ years. The diagnosis of TIDM was based on the criteria for classical TIDM introduced by the WHO in 1999 (8). The protocol and informed consent documents were approved by the Institutional Ethics Committee at Nanjing First Hospital. All of the patients gave written informed consent.

\section{Methods}

Demographic information about the patients was collected, including each patient's age, history of diabetes, BMI, waist circumference, hip circumference, waist-hip ratio, and insulin dose.

Continuous glucose data were obtained using the Medtronic Minimed CGMS Gold (Medtronic Incorporated, Northridge, USA) for at least 3 days after being admitted to the hospital for 2 days. The CGMS induction probe detected the concentration of abdominal subcutaneous interstitial fluid glucose in the subjects. It was connected to a recorder via a cable so that the glucose concentrations could be obtained and recorded every 5 minutes ( $\mathrm{min}$ ). A total of 288 glucose readings were recorded automatically every day for three days, and the range of blood glucose levels recorded was 2.2-22.2 mmol/L (39.6-399.6 mg/dL). Peripheral blood glucose levels were measured at least 4 times per day during the monitoring period to calibrate the CGMS results. The data obtained from the CGMSs included the following parameters: the 24-hour (hr) mean blood glucose level (MBG), the standard deviation of the MBG level (SDBG), the 24-hr mean amplitude of glycemic excursions (MAGE), the percentage time durations $(\%)$ of hyperglycemia (glucose $>13.3$ $\mathrm{mmol} / \mathrm{L})(>239.4 \mathrm{mg} / \mathrm{dL}$ ) and hypoglycemia (glucose $<3.9 \mathrm{mmol} / \mathrm{L})(<70.2 \mathrm{mg} / \mathrm{dL})$, the maximum blood glucose level (Max) and the minimum blood glucose level (Min).

The clinical and biochemical parameters included the following: fasting C-peptide (C-P0), 2-hr postprandial C-peptide (C-P2hr), fasting glucagon (Glu0), 2-hr postprandial glucagon (Glu2hr), HbAlc, anti-GAD or insulin antibodies, blood routine, biochemical indexes, and calculations for fasting glucagon/ fasting C-peptide (Glu0/C-P0) and 2-hr postprandial glucagon/2-hr postprandial C-peptide (Glu2hr/C$\mathrm{P} 2 \mathrm{hr}$ ). Plasma glucose levels were measured using the glucose oxidase method. C-peptide was measured using a chemiluminescent immunometric assay on the Modular Analytics E170 (Roche ${ }^{\circledR}$ Diagnostics GmbH, Mannheim, Germany). Glucagon levels were determined using a quantitative radioimmunoprecipitation assay kit (Beijing North Institute of Biological Technology, China). HbAlc levels were measured using a highperformance liquid chromatography (HPLC) assay (Bio-Rad Laboratories, Inc. CA, USA). The breakfast for the patients' OGTT was $250 \mathrm{ml}$ milk, $0.1 \mathrm{~kg}$ steamed bread meal, and an egg.

\section{Statistical analysis}

For normally distributed data, the means \pm standard deviations (SDs) of the two groups were compared using t-tests. For data that were nonnormally distributed, the medians and interquartile ranges were compared using nonparametric Mann-Whitney U tests. The rates between the two groups were compared using the chisquared test. Significance was defined as $\mathrm{p}<0.05$.

\section{RESULTS}

\section{Patient demographics}

There were no significant differences in the genders and histories of diabetes between the two groups. Ages, BMIs, and waist-to-hip ratios in the FTIDM group were higher than those in the TIDM group ( $\mathrm{p}<0.05$ for all); premeal insulin doses (unit $/ \mathrm{kg}$ / day) were significantly higher in the FTIDM group compared to the TIDM group $(\mathrm{p}<0.05)$. Daily doses of insulin (unit $/ \mathrm{kg} /$ day) and basal doses of insulin (unit $/ \mathrm{kg} /$ day) were similar between the two groups $(\mathrm{p}>0.05)($ Table 1$)$.

\section{Laboratory measurements}

Patients with FT1DM had lower C-P0 and C-P2hr levels and higher Glu2hr, Glu0/C-P0, and Glu2hr/C-P2hr levels than those with T1DM $(\mathrm{p}<0.05)$, suggesting that the regulation of glucagon secretion from $\alpha$ cells is impaired in FTIDM. GAD antibody levels in the FTIDM group were significantly lower than in the TIDM group $(\mathrm{p}<0.05)$. There were no significant differences in HbAlc, INS-Ab, and Glu0 levels between the two groups ( $p>0.05$ for all) (Table 1$)$. 
Blood urea nitrogen (BUN) and alkaline phosphatase levels were significantly higher in the FTIDM group than in the TIDM group $(\mathrm{p}<0.05)$. There were no significant differences in the blood routines (white blood cells, red blood cells, hemoglobin, and platelet count) or biochemical indexes (alanine aminotransferase, albumin, globulin, potassium, sodium, chlorine, cholesterol, triglyceride, high density lipoprotein, glutamine transpeptidase, blood creatinine, urinary albumin, and uric acid) between the two groups ( $\mathrm{p}>0.05$ for all) (Table 1$)$.

Table 1. Clinical and biochemical characteristics

\begin{tabular}{|c|c|c|c|c|}
\hline Groups & FT1DM $(n=10)$ & T1DM $(n=20)$ & $\mathrm{t} / \mathrm{z} / \chi^{2}$ & $\mathbf{P}$ \\
\hline Gender (\%) & $4 / 6$ & $12 / 8$ & 1.071 & 0.442 \\
\hline Age (years) & $43(37.5,54)$ & $35(26.75,38.75)$ & -2.312 & $0.021^{*}$ \\
\hline History (years) & $6(3.25,10.5)$ & $9.5(2.25,17)$ & -0.596 & 0.551 \\
\hline Body mass index (kg/m²) & $23.01 \pm 2.64$ & $20.98 \pm 2.01$ & 2.348 & $0.026^{\star}$ \\
\hline Wasit to hip ratio (\%) & $0.98 \pm 0.04$ & $0.93 \pm 0.06$ & 2.454 & $0.021^{*}$ \\
\hline The basal dosages of insulin ( $\mathrm{u} / \mathrm{kg} /$ day) & $0.24 \pm 0.08$ & $0.27 \pm 0.15$ & -0.582 & 0.565 \\
\hline The pre-meal dosages of insulin ( $\mathrm{u} / \mathrm{kg} / \mathrm{day})$ & $0.31(0.26,0.61)$ & $0.24(0.17,0.31)$ & -2.268 & $0.023^{*}$ \\
\hline The daily dosages of Insulin (u/kg/day) & $0.63 \pm 0.20$ & $0.52 \pm 0.18$ & 1.657 & 0.109 \\
\hline Fasting C-peptide ( $\mu \mathrm{g} / \mathrm{L})$ & $0.01(0.01,0.04)$ & $0.07(0.01,0.20)$ & -2.227 & $0.026^{\star}$ \\
\hline 2-hr postprandial C-peptide ( $\mu \mathrm{g} / \mathrm{L})$ & $0.01(0.01,0.04)$ & $0.11(0.02,0.52)$ & -2.778 & $0.005^{\star *}$ \\
\hline Fasting glucagon (ng/L) & $156.06 \pm 43.86$ & $136.19 \pm 28.03$ & 1.512 & 0.142 \\
\hline 2-hr postprandial glucagon (ng/L) & $208.74(166.39,271.73)$ & $165.31(144.93,200.39)$ & -2.202 & $0.028^{*}$ \\
\hline Fasting glucagon/Fasting C-peptide (\%) & $13271.00(5193.94,18294.25)$ & $2227.86(627.63,13705.75)$ & -2.024 & $0.043^{*}$ \\
\hline 2-hr postprandial glucagon/2-hr postprandial C-peptide (\%) & $17595.50(9020.94,26907.00)$ & $1339.37(291.14,7466.25)$ & -3.08 & $0.002^{\star \star}$ \\
\hline Glycosylated hemoglobin (\%) & $8.03 \pm 1.31$ & $9.70 \pm 2.65$ & -1.864 & $0.073^{\star}$ \\
\hline Anti-GAD antibodies (\%) & $5.48(4.67,7.21)$ & $13.40(9.36,23.03)$ & -3.696 & $0.000^{\star *}$ \\
\hline Anti-Insulin antibodies (\%) & $3.37(2.86,8.26)$ & $6.09(2.95,11.62)$ & -1.16 & 0.246 \\
\hline White blood cells ( $\left.{ }^{*} 10^{9} / L\right)$ & $6.02 \pm 3.51$ & $6.88 \pm 2.83$ & -0.728 & 0.473 \\
\hline Red blood cells $\left({ }^{*} 10^{12} / \mathrm{L}\right)$ & $4.12 \pm 0.44$ & $4.42 \pm 0.49$ & -1.632 & 0.114 \\
\hline Platelet count ( $\left.{ }^{*} 10^{9} / \mathrm{L}\right)$ & $156.8 \pm 56.13$ & $196.05 \pm 63.82$ & -1.649 & 0.110 \\
\hline Haemoglobin (g/L) & $131 \pm 9.24$ & $134.85 \pm 18.51$ & -0.617 & 0.543 \\
\hline Alanine aminotransferase (U/L) & $25.00(16.50,38.75)$ & $19.00(11.00,24.75)$ & -1.652 & 0.099 \\
\hline Albumin (g/L) & $41.86 \pm 9.67$ & $42.63 \pm 3.59$ & -0.317 & 0.754 \\
\hline Globulin (g/L) & $25.32 \pm 3.76$ & $25.49 \pm 4.77$ & -0.098 & 0.922 \\
\hline Potassium (mmol/L) & $4.05(3.71,4.27)$ & $4.42(3.79,4.74)$ & -1.651 & 0.099 \\
\hline Sodium (mmol/L) & $141.05 \pm 4.87$ & $138.94 \pm 4$ & 1.269 & 0.215 \\
\hline Chloride (mmol/L) & $102.97 \pm 5.59$ & $102.15 \pm 4.08$ & 0.461 & 0.648 \\
\hline Cholesterol (mmol/L) & $4.66 \pm 0.77$ & $5.26 \pm 0.94$ & -1.736 & 0.094 \\
\hline Triglyceride (mmol/L) & $1.12 \pm 0.78$ & $1.03 \pm 0.71$ & 0.315 & 0.755 \\
\hline HDL-cholesterol (mmol/L) & $1.5 \pm 0.51$ & $1.77 \pm 0.53$ & -1.344 & 0.190 \\
\hline Glutamyl transpeptidase (U/L) & $18.5(12.00,27.75)$ & $15.50(12.00,24.50)$ & -0.794 & 0.427 \\
\hline Alkaline phosphatase (U/L) & $98.70 \pm 25.27$ & $72.25 \pm 18.00$ & 3.312 & $0.003^{\star *}$ \\
\hline Urinary albumin (\%) & $3 / 7$ & $2 / 18$ & 1.92 & 0.300 \\
\hline Blood urea nitrogen (mmol/L) & $6.66(6.18,10.65)$ & $5.50(3.97,6.55)$ & -2.376 & $0.017^{\star}$ \\
\hline Uric Acid (umol/L) & $375.50(141.75,519.25)$ & $304(253,391)$ & -0.311 & 0.755 \\
\hline Blood creatinine (mmol/L) & $86.00(62.75,117.50)$ & $68.7(54.08,74.95)$ & -1.716 & 0.086 \\
\hline
\end{tabular}

Normally distributed data was compared using t-test. Non-normally distributed data was compared using the nonparametric Mann-Whitney test.

The rates between the two groups were compared using the chi-square test. ${ }^{*}=p<0.05,{ }^{* *}=p<0.01$. 


\section{CGMS results}

The CGMS data showed that the percentage of blood glucose levels $<3.9 \mathrm{mmol} / \mathrm{L}$ was higher in the FTIDM group than in the TIDM group $(\mathrm{p}<0.05)$. The Min blood glucose level in the FTIDM group was lower than the Min in the TIDM group $(\mathrm{p}<0.05)$ (Table 2).

Other CGMS parameters (MBG, SDBG, MAGE, Max and the percentage of blood glucose levels > $13.9 \mathrm{mmol} / \mathrm{L}$ ) were similar between the two groups ( $\mathrm{p}>0.05$ for all).

\section{DISCUSSION}

In the present study, CGMS data showed that patients in the FTIDM group had a higher risk of hypoglycemia at the stable stage, worse destruction of pancreatic beta cells, and more severe dysfunction of the islet alpha cells than patients with TIDM. Renal dysfunction was more commonly found in patients with FTIDM. Other CGMS parameters did not differ significantly between the two groups.

There were no significant differences found in $\mathrm{MBG}$, SDBG, MAGE and Max levels between the FTIDM and TIDM patients. These results indicate that blood glucose fluctuations were similar in the FTIDM and TIDM groups. However, the percentage of blood glucose levels $<3.9 \mathrm{mmol} / \mathrm{L}$ was higher and the Min blood glucose level was lower in the FTIDM group compared to the TIDM group ( $\mathrm{p}<0.05$ for both). These findings suggest that patients with FTIDM have a higher risk of hypoglycemia compared to patients with TIDM. A 5-year follow-up study of 41 patients with FTIDM performed by Murase and cols. showed that blood glucose fluctuations (measured using 7-point blood glucose monitoring) and the frequencies of severe hypoglycemia were higher in a group of FTIDM patients than in a group of TIDM patients (9). The results of the current study partially agree with this, as we only found that patients with FTIDM had a higher risk of hypoglycemia compared to patients with TIDM, which was indicated by the higher percentage of blood glucose levels $<3.9 \mathrm{mmol} / \mathrm{L}$ and the lower minimum blood glucose levels in the FTIDM group. However, we did not find significant differences among other indexes for blood glucose fluctuations between the two groups. This difference may be associated with the methods that were used to monitor blood glucose levels. We used CGMSs to monitor blood glucose levels, which are more accurate and occur in real-time (10). We also noticed that our patients with FTIDM were older and had higher BMIs and waist-to-hip ratios than the TIDM patients. The finding of higher BMIs in patients with FTIDM compared with those with TIDM was consistent with results of previous studies (2,11-13). The higher BMIs in patients with FTIDM compared with those with TIDM may be due to the rapid onset and short duration of FTIDM, which does not reduce body weight significantly. However, beta cell function was severely damaged, which led to lower C-peptide levels in patients with FTIDM.

Furthermore, we observed that premeal doses of insulin (unit $/ \mathrm{kg} /$ day) were significantly higher in the FTIDM group than in the TIDM group. However, while the total daily doses of insulin were higher in the FTIDM group, there was no significant difference between the two groups. One possible reason for

Table 2. CGMS data

\begin{tabular}{|c|c|c|c|c|c|}
\hline Indexes & FT1DM $(n=10)$ & T1DM $(n=20)$ & $\begin{array}{l}\text { 95\% confidence } \\
\text { interval }\end{array}$ & $t / z$ & $\mathbf{P}$ \\
\hline Mean blood glucose (mmol/L) & $8.86 \pm 3.21$ & $11.39 \pm 3.36$ & $(-5.1637,0.0917)$ & -1.977 & 0.058 \\
\hline Standard deviation of blood glucose (mmol/L) & $3.47 \pm 1.07$ & $3.12 \pm 1.25$ & $(-0.5912,1.3012)$ & 0.769 & 0.449 \\
\hline Mean amplitude glycemic excursions (mmol/L) & $9.56 \pm 2.77$ & $7.76 \pm 3.72$ & $(-0.9354,4.5213)$ & 1.346 & 0.189 \\
\hline Percentage of time above $13.9\left(\mathrm{PT}_{1}\right)$ & $8.00(2.00,49.00)$ & $15.50(1.50,57.50)$ & l & -0.529 & 0.597 \\
\hline AUC $>13.9 \mathrm{mmol} / \mathrm{L}$ & $0.10(0,1.15)$ & $0.325(0,2.05)$ & / & -0.880 & 0.379 \\
\hline Percentage of time below 3.9 (PT2) & $16.00(0,34.50)$ & $0(0,0)$ & / & -2.979 & $0.003^{\star \star}$ \\
\hline AUC $<3.9 \mathrm{mmol} / \mathrm{L}$ & $0.10(0,0.20)$ & $0(0,0)$ & / & -3.388 & $0.001^{\star *}$ \\
\hline The maximum (mmol/L) & $16.16 \pm 3.38$ & $17.27 \pm 4.40$ & $(-4.3635,2.1415)$ & -0.700 & 0.490 \\
\hline The minimum (mmol/L) & $2.40(2.20,5.03)$ & $5.15(3.83,6.55)$ & I & -2.360 & $0.018^{\star}$ \\
\hline
\end{tabular}

AUC $=$ Area under curve. Normally distributed data was compared using t-test. Non- normally distributed data was compared using the nonparametric Mann-Whitney test. The rates between the two groups were compared using the chi-square test. ${ }^{\star}=p<0.05,{ }^{\star \star}{ }^{\star}=p<0.01$. 
the pre-meal doses of insulin may be related to the more severely damaged islet function in patients with FTIDM, i.e., the poor function of islet beta cells and the dysfunction of islet alpha cells, resulting in a need for more insulin to reduce the postprandial blood glucose level. Imagawa and cols. found that daily doses of insulin were significantly higher in 161 FTIDM patients than in TIDM patients in follow-ups at 3,6, and 12 months (11). This is different from the results of our study, and this difference may be related to the sample size, as we only studied 10 patients whose total daily doses of insulin had a tendency to be higher $(0.63$ $\pm 0.20 \mathrm{u} / \mathrm{kg} /$ day $)$ than those of TIDM patients $(0.52$ $\pm 0.18 \mathrm{u} / \mathrm{kg} /$ day, $\mathrm{p}=0.109$ ).

Islet function was more severely damaged in FT1DM patients, as they had lower C-P0 and C-P2hr levels and higher Glu2hr, Glu0/C-P0, and Glu2hr/ C-P2hr levels compared with TIDM patients $(\mathrm{p}<0.05)$. Though the pathogenesis of FTlDM is currently unclear, it may be mediated by a variety of factors: viral infections, pregnancy, drugs, etc., in addition to autoimmune and genetic factors that can trigger an accelerated immune reaction and promote massive beta-cell death in genetically susceptible individuals. In contrast, a small number of islet cells may still be viable in TIDM patients at the onset of the disease $(2,6,14,15)$. Zheng and cols. proposed that the appearance of a low titer of autoantibodies was likely caused by the viral infection destroying the islet cells (3). Shibasaki and cols. suggested that FTIDM is associated with viral infections and is affected by the patient's genetic background (16). However, Imagawa and cols. found that although GAD-AB, INS-AB, and other related antibodies appeared in patients with FTIDM, they were in low antibody titers and appeared in a short-term national survey (11). Studies have suggested that intestinal viruses and chemokines not only damage the islet beta cells but also accelerate the autoimmune reaction, which destroys the remaining islet beta cells $(17,18)$. Our study found that the GAD-AB was significantly lower in the FTIDM group than in the TIDM group, while the INS-AB titer findings were similar between the two groups. This is consistent with previous reports $(3,9)$.

Patients with FT1DM had lower C-P0 and C-P2hr levels than patients with TIDM in our study, which could be related to the stage of onset of disease $(2,6)$. This suggests that the function of islet beta cells in FTIDM was rapidly damaged from the onset of the disease, progressively declined, and could still be declining in TIDM after a few years. Meanwhile, patients with FTIDM had higher Glu2hr, Glu0/C-P0, and Glu2hr/C-P2hr levels than those with TlDM, suggesting that glucagon secretion was also damaged in the FTIDM group compared to the TIDM group.

The results of our study were consistent with those of the studies of Liu and Fany $(5,19)$, who more clearly showed a regulating function disorder in the islet alpha cells in an FTIDM group. Sayama and cols. showed through morphological observations that islet alpha and beta cells were destroyed in FTIDM patients, while islet beta cells were destroyed and islet alpha cells remained functional in TIDM patients at the early stage of the disease (20). Fan and cols. followed 6 FTIDM patients for 9-72 months and reported that fasting and postprandial C-P2hr levels were close to levels requiring hospitalization at the acute stage of the disease, which suggested that the islet beta cells were completely and irreversibly destroyed (19). A report by Huang and cols. showed that the function of the islet beta cells in 2 FTIDM patients did not improve after 7 months of follow up (21).

There were no significant differences in white blood cells, neutrophils, alanine, aspartate, potassium, cholesterol, high density lipoprotein, creatinine, and glutamine transpeptidase levels between the two groups. However, cholesterol and high-density lipoprotein levels were lower and white blood cells, neutrophils, alanine, aspartate, potassium, creatinine, and glutamine transpeptidase levels were generally higher in FTIDM patients at the acute stage [2]. This suggests that FTIDM caused not only metabolic and biochemical disorders but also tissue dysfunction or injuries, including in the liver and kidney, at the acute stage. Though metabolic and some biochemical indicators could return to the normal range after treatment at the stable stage, the liver and kidney injuries are irreversible, as indicated by the BUN and alkaline phosphatase levels being significantly higher in the FTIDM group compared to the TIDM group $(\mathrm{P}<0.05)$. BUN is an important indicator of renal function, while alkaline phosphatase is mainly derived from the liver, bone, and kidneys; it indicates early kidney damage (22-24). Compromised kidney function following the acute stage of FTIDM may be caused by large blood glucose fluctuations, immune reactions, inflammation, and severe hypoglycemia.

In conclusion, the present study suggests that patients with FTIDM at the stable stage and patients 
with TIDM had similar blood glucose fluctuation ranges. However, patients with FTIDM had a higher proportion of hypoglycemic levels, severely damaged pancreatic beta cells and more severe dysfunction of the islet alpha cells and kidney compared to patients with TIDM.

Compliance with Ethical Standards: all procedures performed in studies involving human participants were completed in accordance with the ethical standards of the institutional and/or national research committee and the 1964 Helsinki Declaration and its later amendments or comparable ethical standards.

Acknowledgements: we thank all patients and control subjects for participating in this study.

Funding: this study was funded by the National Natural Science Foundation of China (NO. 81500606), the Jiangsu Provincial Department of Science and Technology Project (No. BL2014010) and the National Science Foundation of Jiangsu Province (No. SBK2015042970).

Disclosure: no potential conflict of interest relevant to this article was reported.

\section{REFERENCES}

1. Imagawa A, Hanafusa T, Miyagawa J, Matsuzawa Y. A novel subtype of type 1 diabetes mellitus characterized by a rapid onset and an absence of diabetes-related antibodies. Osaka IDDM Study Group. N Engl J Med. 2000;342(5):301-7.

2. Su XF, Fu LY, Wu JD, Xu XH, Li HQ, Sun R, et al. Fulminant type 1 diabetes mellitus: a study of nine cases. Diabetes Technol Ther. 2012;14(4):325-9.

3. Zheng C, Zhou Z, Yang L, Lin J, Huang G, Li X, et al. Fulminant type 1 diabetes mellitus exhibits distinct clinical and autoimmunity features from classical type 1 diabetes mellitus in Chinese. Diabetes Metab Res Rev. 2011;27(1):70-8.

4. Cho YM, Kim JT, Ko KS, Koo BK, Yang SW, Park MH, et al. Fulminant type 1 diabetes in Korea: high prevalence among patients with adult-onset type 1 diabetes. Diabetologia. 2007;50(11):2276-9.

5. Liu L, Mao J, Lu Z, Yan X, YeY, Jiang F. Clinical analysis of fulminant type 1 diabetes in China and comparison with a nationwide survey in Japan. Annales d'endocrinologie. 2013;74:36-9.

6. Imagawa A, Hanafusa T. Fulminant type 1 diabetes--an important subtype in East Asia. Diabetes Metab Res Rev. 2011;27(8):959-64.

7. Imagawa A, Hanafusa T, Awata T, Ikegami H, Uchigata Y, Osawa H, et al. Report of the Committee of the Japan Diabetes Society on the Research of Fulminant and Acute-onset Type 1 Diabetes Mellitus: New diagnostic criteria of fulminant type 1 diabetes mellitus (2012). J Diabetes Investig. 2012;3(6):536-9.

8. Koga M, Shimizu I, Murai J, Saito H, Kasayama S, Kobayashi T, et al. The glycated albumin to $\mathrm{HbA} 1 \mathrm{c}$ ratio is elevated in patients with fulminant type 1 diabetes mellitus with onset during pregnancy. J Med Invest. 2013;60(1-2):41-5.

9. Murase $Y$, Imagawa A, Hanafusa T, Iwahashi H, Uchigata $Y$, Kanatsuka $A$, et al. Fulminant type 1 diabetes as a high risk group for diabetic microangiopathy--a nationwide 5-year-study in Japan. Diabetologia. 2007;50(3):531-7.

10. Zhou J, Lv X, MuY, Wang X, Li J, Zhang X, et al. The accuracy and efficacy of real-time continuous glucose monitoring sensor in Chinese diabetes patients: a multicenter study. Diabetes Technol Ther. 2012;14(8):710-8.

11. Imagawa A, Hanafusa T, Uchigata Y, Kanatsuka A, Kawasaki E, KobayashiT, et al. Fulminant type 1 diabetes: a nationwide survey in Japan. Diabetes Care. 2003;26(8):2345-52.

12. Su XF, Wu JD, Li Q, et al. Clinical features of 6 patients with fulminant type 1 diabetes mellitus. Chinese Journal of Diabetes Mellitus. 2010;2(5):329-33.

13. Luo S, Zhang Z, Li X, Yang L, Lin J, Yan X, et al. Fulminant type 1 diabetes: a collaborative clinical cases investigation in China. Acta Diabetol. 2013;50(1):53-9.

14. Kim HJ, Kim HS, Hahm JR, Jung JH, Kim SK, Lee SM, et al. The first Vietnamese patient with fulminant type 1 diabetes mellitus. Intern Med. 2012;51(17):2361-3.

15. Tsutsumi $C$, Imagawa A, Ikegami $H$, Makino $H$, Kobayashi $T$, Hanafusa T; Japan Diabetes Society Committee on Type 1 Diabetes Mellitus Research. Class II HLA genotype in fulminant type 1 diabetes: A nationwide survey with reference to glutamic acid decarboxylase antibodies. J Diabetes Investig. 2012;3(1):62-9.

16. Shibasaki S, Imagawa A, Hanafusa T. Fulminant type 1 diabetes mellitus: a new class of type 1 diabetes. Adv Exp Med Biol. 2012;771:20-3.

17. Aida K, Nishida Y, Tanaka S, et al. RIG-I- and MDA5-initiated innate immunity linked with adaptive immunity accelerates betacell death in fulminant type 1 diabetes. Diabetes. 2011;60:884-9.

18. Tanaka S, Nishida Y, Aida K, Maruyama T, Shimada A, Suzuki M, et al. Enterovirus infection, CXC chemokine ligand 10 (CXCL10), and CXCR3 circuit: a mechanism of accelerated beta-cell failure in fulminant type 1 diabetes. Diabetes. 2009;58(10):2285-91.

19. Fany YJ, Lu B, Yang JL. clinical analysis of six cases of fulminant type 1 diabetes mellitus. Chin J Diabetes. 2014;22(5):542-4.

20. Sayama K, Imagawa A, Okita K, Uno S, Moriwaki M, Kozawa J, et al. Pancreatic beta and alpha cells are both decreased in patients with fulminant type 1 diabetes: a morphometrical assessment. Diabetologia. 2005;48(8):1560-4.

21. Huang HB, Gong $X Y$, Lin LX, Chen G. Approach to the patient with fulminant type I diabetes. Chin J Endocrinol Metab. 2014;30(1): 83-6.

22. Tuo SX, Qian YX, Yang L, Zhang YQ, Yu SQ. Relationship between Bone Mineral Density and Serum Bone Metabolism Biomarkers in Patients with Chronic Kidney Disease. Chin Med J. 2013;16(10):3422-4.

23. Li YP, Liao YH, Pan L, Ding WJ. Bone - kidney - parathyroid Axes Related Cytokines and Its Influencing Factors in Patients with Chronic Kidney Disease. 2014;19:2224-7.

24. Feng Q, Deng DY, Yuan WL, et al. Analysis of serum bone metabolism biochemical index of the Chronic Kidney Disease (CKD) 4-5 patients between diabetic nephropathy and non-diabetic nephropathy. Int J Lab Med. 2012;33(24):2992-3. 